\title{
Towards Practical Non-interactive Public Key Cryptosystems Using Non-maximal Imaginary Quadratic Orders (Extended Abstract)
}

\author{
Detlef Hühnlein ${ }^{1}$, Michael J. Jacobson, Jr. ${ }^{2}$, and Damian Weber ${ }^{3}$ \\ 1 Secunet Security Networks AG, \\ Mergenthalerallee 77-81, D-65760 Eschborn, Germany \\ huehnlein@secunet.de \\ 2 Centre for Applied Cryptographic Research, \\ University of Waterloo, \\ Waterloo, Ontario, Canada, N2L 3G1 \\ mjjacobs@cacr.math. uwaterloo.ca \\ 3 Märkische Fachhochschule Iserlohn und Hagen, \\ Haldener Str. 182, D-58095 Hagen, Germany \\ weber@mfh-iserlohn. de
}

\begin{abstract}
We present a new non-interactive public key distribution system based on the class group of a non-maximal imaginary quadratic order $C l\left(\Delta_{p}\right)$. The main advantage of our system over earlier proposals based on $(\mathbb{Z} / n \mathbb{Z})^{*} 1921$ is that embedding id information into group elements in a cyclic subgroup of the class group is easy (straight-forward embedding into prime ideals suffices) and secure, since the entire class group is cyclic with very high probability.

In order to compute discrete logarithms in the class group, the KGC needs to know the prime factorization of $\Delta_{p}=\Delta_{1} p^{2}$. We present an algorithm for computing discrete logarithms in $C l\left(\Delta_{p}\right)$ by reducing the problem to computing discrete logarithms in $C l\left(\Delta_{1}\right)$ and either $\mathbb{F}_{p}^{*}$ or $\mathbb{F}_{p^{2}}^{*}$. We prove that a similar reduction works for arbitrary non-maximal orders, and that it has polynomial complexity if the factorization of the conductor is known.
\end{abstract}

Keywords: discrete logarithm, non-maximal imaginary quadratic order, non-interactive cryptography, identity based cryptosystem

\section{Introduction}

Public-key cryptography is undoubtedly one of the core techniques used to enable authentic, non-repudiable and confidential communication. However, a general problem inherent in public-key systems is that one needs to ensure the authenticity of a given public key. The most common way to solve this problem is to introduce a trusted third party, called a Certification Authority (CA), which is-

D.R. Stinson and S. Tavares (Eds.): SAC 2000, LNCS 2012, pp. 275-287, 2001.

(C) Springer-Verlag Berlin Heidelberg 2001 
sues certificates for public key@ 1 . While this approach is widely used in practice, it would be desirable to have an immediate binding between an identity $I D_{B}$ and its corresponding public key $\mathfrak{b}$, which allows one to avoid the tedious verification of certificates. This leads to the notion of identity based cryptosystems.

Although the paradigm of identity based cryptography was already introduced by Shamir in 1984 [23, it seems that Maurer and Yacobi [19] were the first to propose a non-interactive identity based public key cryptosystem in which Bob's public key $\mathfrak{b}$ can be derived efficiently, solely from his public identity information $I D_{B}$, by computing a publicly-known embedding function $\mathfrak{b}=f\left(I D_{B}\right)$. The main idea is to use an (ideally cyclic) group $G$ (generated by $\mathfrak{g}$ ) in which exponentiation is not only a one-way-function but a trapdoor-one-way-function. The key generation center (KGC), a trusted third party responsible for distributing the private keys, knows the trapdoor information and hence is able to compute discrete logarithms in $G$. Thus, the KGC computes Bob's private key $b$ such that $\mathfrak{g}^{b}=\mathfrak{b}=f\left(I D_{B}\right)$. The KGC hands over the secret key $b$ to Bob, who can use this key in a conventional ElGamal- or Diffie-Hellman setup. As soon as all users are equipped with their corresponding secret key, the KGC can destroy the trapdoor-information and may cease to exist.

Maurer and Yacobi's initial proposal was to set up a discrete logarithm based system in $G=(\mathbb{Z} / n \mathbb{Z})^{*}$, where $n=p_{1} \cdots p_{r}, p_{i}$ prime, such that only the KGC, which knows the factorization of $n$, is able to compute discrete logarithms in $G$. However, this approach has a number of drawbacks which render such a scheme impractical [20[18]17].

In this paper, we show that using the class group $C l\left(\Delta_{p}\right)$ of a non-maximal imaginary quadratic order is much better suited for this purpose. As in the original scheme, the KGC knows trapdoor information (the prime factorization of $\Delta_{p}$ ) which enables it to compute discrete logarithms, while for anybody else the discrete logarithm problem (DLP) is assumed to be intractable. We generalize the recent result from [12, valid for the very special case of totally non-maximal orders with prime discriminant, to arbitrary non-maximal imaginary quadratic orders. The resulting algorithm reduces the problem of discrete logarithm computation in the class group of a non-maximal order to computing discrete logarithms in the much smaller class group of the corresponding maximal order and a small number of finite fields. Only the KGC, which knows the factorization of $\Delta_{p}$, can perform this reduction.

As noted above there are a few advantages to our approach. Unlike the case of $(\mathbb{Z} / n \mathbb{Z})^{*}$, it is heuristically easy to find class groups $C l\left(\Delta_{p}\right)$ which are $c y c l i c$, and hence the embedding of an identity $I D_{B}$ into a group element $\mathfrak{b}$, for which the discrete logarithm exists, is straightforward. As the results from [20[18] demonstrate, it seems to be no trivial task to find an embedding into a subgroup of $(\mathbb{Z} / n \mathbb{Z})^{*}$ which does not facilitate factoring $n$. In fact, the only secure embedding method for $(\mathbb{Z} / n \mathbb{Z})^{*}$ seems to restrict $n$ to having only two large prime factors $p_{1}$

\footnotetext{
${ }^{1}$ We assume throughout this work that Alice (A) wants to encrypt a message $m \in$ $\mathbb{Z}_{>0}$ intended for Bob (B). We denote Bob's unique identity, for example his emailaddress, by $I D_{B}$ and his public key by $\mathfrak{b}$
} 
and $p_{2}$, and the workload for the KGC is consequently very high. Furthermore, since one chooses $p_{i}-1$ smooth and uses Pohlig-Hellman's simplification together with Shank's Baby-Step Giant-Step algorithm, the time needed for generating $k$ user keys is proportional to $k$.

In contrast, we use two different subexponential algorithms for the key generation. After the initial computation of relations over the factor bases, the workload for each individual key generation is very modest. For the computation of discrete logarithms in the class group of the maximal order, $C l\left(\Delta_{1}\right)$, we use an analogue of the Self-Initializing Quadratic Sieve (SIQS) factoring algorithm 14,13 and for the computation of discrete logarithms in $\mathbb{F}_{p}^{*}$ we use the Special Number Field Sieve, which recently was used for the solution of McCurley's challenge [24].

This paper is organized as follows: in Section 2 we provide the necessary background and notation for non-maximal imaginary quadratic orders. The next section contains the discrete logarithm algorithm for arbitrary non-maximal imaginary quadratic orders, and in Section 4 we present our new non-interactive public key cryptosystem. In order to save space, the proofs of most results have been omitted. These proofs, as well as computational results, will be given in the full paper [10].

\section{Non-maximal Imaginary Quadratic Orders}

The basic notions of imaginary quadratic number fields can be found in 112 . For a more comprehensive treatment of the relationship between maximal and non-maximal orders we refer to 5912 .

Let $\mathcal{O}_{\Delta_{f}}$ denote the non-maximal quadratic order of discriminant $\Delta_{f}=\Delta_{1} f^{2}$ with conductor $f$, and let $\mathcal{O}_{\Delta_{1}}$ denote the corresponding maximal order. When the conductor is prime, we will use $\mathcal{O}_{\Delta_{p}}$ and $\Delta_{p}$. By $C l\left(\Delta_{f}\right)$ and $C l\left(\Delta_{1}\right)$ we denote the ideal class groups of $\mathcal{O}_{\Delta_{f}}$ and $\mathcal{O}_{\Delta_{1}}$, respectively. The class numbers $h\left(\Delta_{f}\right)$ and $h\left(\Delta_{1}\right)$ are the orders of these groups. Lower-case Gothic letters $\mathfrak{a}, \mathfrak{b}, \ldots$ denote ideals in $\mathcal{O}_{\Delta_{f}}$ and upper-case Gothic letters denote ideals in $\mathcal{O}_{\Delta_{1}}$. Ideal equivalence is denoted by $\mathfrak{a} \sim \mathfrak{b}$, and the class of all ideals equivalent to $\mathfrak{a}$ is denoted by $[\mathfrak{a}]$. Throughout, we will use $\Delta$ without subscript to denote the discriminant of an arbitrary quadratic order, maximal or non-maximal.

Our cryptosystem makes use of the relationship between a non-maximal order of conductor $f$ and its corresponding maximal order. Any non-maximal order can be represented as $\mathcal{O}_{\Delta_{f}}=\mathbb{Z}+f \mathcal{O}_{\Delta_{1}}$. If $h\left(\Delta_{1}\right)=1$, then $\mathcal{O}_{\Delta_{f}}$ is called a totally non-maximal order. An integral ideal $\mathfrak{a}$ is called prime to $f$ if $\operatorname{gcd}(\mathcal{N}(\mathfrak{a}), f)=1$. It is well-known that all $\mathcal{O}_{\Delta_{f}}$-ideals prime to the conductor are invertible, and in every ideal equivalence class there is an ideal which is prime to any given number. We denote the principal $\mathcal{O}_{\Delta_{f}}$-ideals prime to $f$ by $\mathcal{P}_{\Delta_{f}}(f)$ and all fractional ideals which are prime to $f$ by $\mathcal{I}_{\Delta_{f}}(f)$. There is an isomorphism

$$
\mathcal{I}_{\Delta_{f}}(f) / \mathcal{P}_{\Delta_{f}}(f) \simeq \mathcal{I}_{\Delta_{f}} / \mathcal{P}_{\Delta_{f}}=C l\left(\Delta_{f}\right)
$$


so we can "ignore" the ideals which are not prime to the conductor if we are only interested in the class group $C l\left(\Delta_{f}\right)$.

There is an isomorphism between the group of $\mathcal{O}_{\Delta_{f}}$-ideals which are prime to $f$ and the group of $\mathcal{O}_{\Delta_{1}}$-ideals which are prime to $f$, denoted by $\mathcal{I}_{\Delta_{f}}(f)$, and $\mathcal{I}_{\Delta_{1}}(f)$, respectively.

Proposition 1. Let $\mathcal{O}_{\Delta_{f}}$ be an order of conductor $f$ in an imaginary quadratic field $\mathbb{Q}\left(\sqrt{\Delta_{1}}\right)$ with maximal order $\mathcal{O}_{\Delta_{1}}$.

(i.) If $\mathfrak{A} \in \mathcal{I}_{\Delta_{1}}(f)$, then $\mathfrak{a}=\mathfrak{A} \cap \mathcal{O}_{\Delta_{f}} \in \mathcal{I}_{\Delta_{f}}(f)$ and $\mathcal{N}(\mathfrak{A})=\mathcal{N}(\mathfrak{a})$.

(ii.) If $\mathfrak{a} \in \mathcal{I}_{\Delta_{f}}(f)$, then $\mathfrak{A}=\mathfrak{a} \mathcal{O}_{\Delta_{1}} \in \mathcal{I}_{\Delta_{1}}(f)$ and $\mathcal{N}(\mathfrak{a})=\mathcal{N}(\mathfrak{A})$.

(iii.) The map $\varphi: \mathfrak{A} \mapsto \mathfrak{A} \cap \mathcal{O}_{\Delta_{f}}$ induces an isomorphism $\mathcal{I}_{\Delta_{1}}(f) \stackrel{\sim}{\rightarrow} \mathcal{I}_{\Delta_{f}}(f)$.

The inverse of this map is $\varphi^{-1}: \mathfrak{a} \mapsto \mathfrak{a} \mathcal{O}_{\Delta_{1}}$.

Thus we are able to switch to and from ideals in the maximal and non-maximal orders via the map $\varphi$. The algorithms $\operatorname{GoToMax} \operatorname{Order}(\mathfrak{a}, f)$ to compute $\varphi^{-1}$ and GoToNonMaxOrder $(\mathfrak{A}, f)$ to compute $\varphi$ can be found in [9]. If $\mathfrak{a}=a \mathbb{Z}+$ $\frac{b+\sqrt{\Delta_{f}}}{2} \mathbb{Z}=(a, b)$ and $\mathfrak{A}=A \mathbb{Z}+\frac{B+\sqrt{\Delta_{1}}}{2} \mathbb{Z}=(A, B)$ are reduced ideals, then these algorithms need $O\left(\log \left(\left|\Delta_{1}\right|\right)^{2}\right)$ and $O\left(\log \left(\left|\Delta_{f}\right|\right)^{2}\right)$ bit-operations respectively.

It is important to note that the isomorphism $\varphi$ is between the ideal groups $\mathcal{I}_{\Delta_{1}}(f)$ and $\mathcal{I}_{\Delta_{f}}(f)$ and not the class groups. If, for $\mathfrak{A}, \mathfrak{B} \in \mathcal{I}_{\Delta_{1}}(f)$ we have $\mathfrak{A} \sim$ $\mathfrak{B}$, it is not necessarily true that $\varphi(\mathfrak{A}) \sim \varphi(\mathfrak{B})$. On the other hand, equivalence does hold under $\varphi^{-1}$. More precisely we have the following:

Proposition 2. The isomorphism $\varphi^{-1}$ induces a surjective homomorphism $\phi_{C l}^{-1}: C l\left(\Delta_{f}\right) \rightarrow C l\left(\Delta_{1}\right)$, where $[\mathfrak{a}] \mapsto\left[\varphi^{-1}(\mathfrak{a})\right]$.

We now focus on the kernel $\operatorname{Ker}\left(\phi_{C l}^{-1}\right)$ of this map, which will turn out to be of central importance for the computation of discrete logarithms in $C l\left(\Delta_{f}\right)$. In particular, we will need to compute discrete logarithms of elements in $\operatorname{Ker}\left(\phi_{C l}^{-1}\right)$. Representing elements of $\operatorname{Ker}\left(\phi_{C l}^{-1}\right)$ as ideal equivalence classes is completely inadequate for this purpose since we would have to compute discrete logarithms in $C l\left(\Delta_{f}\right)$. Fortunately, there exists an alternative representation which allows us to reduce the problem of computing discrete logarithms in $\operatorname{Ker}\left(\phi_{C l}^{-1}\right)$ to that in a small number of finite fields.

Proposition 3. The map $\psi:\left(\mathcal{O}_{\Delta_{1}} / f \mathcal{O}_{\Delta_{1}}\right)^{*} \rightarrow \operatorname{Ker}\left(\phi_{C l}^{-1}\right),[\alpha] \mapsto\left[\varphi\left(\alpha \mathcal{O}_{\Delta_{1}}\right)\right]$, is a surjective homomorphism.

This homomorphism suggests the following representation for ideal classes in the kernel:

Definition 1. Let $[\alpha]=[x+y \omega] \in\left(\mathcal{O}_{\Delta_{1}} / f \mathcal{O}_{\Delta_{1}}\right)^{*}$ and let $\mathfrak{a} \sim \varphi\left(\alpha \mathcal{O}_{\Delta_{1}}\right)$ be a reduced $\mathcal{O}_{\Delta_{f}}$-ideal whose equivalence class lies in $\operatorname{Ker}\left(\phi_{C l}^{-1}\right)$. Then the pair $(x, y)$ is called a generator representation for the equivalence class $[\mathfrak{a}]$. 
Remark 1. Note that this generator representation $(x, y)$ for the class of $\mathfrak{a}$ is not unique. It is easy to see that $(k x, k y), k \in(\mathbb{Z} / f \mathbb{Z})^{*}$, is also a generator representation for the class of $\mathfrak{a}$. This means that we have $\mathfrak{a} \sim \varphi\left((x+y \omega) \mathcal{O}_{\Delta_{1}}\right) \sim$ $\varphi\left((k x+k y \omega) \mathcal{O}_{\Delta_{1}}\right)$. In other words, $\operatorname{Ker}\left(\phi_{C l}^{-1}\right) \cong\left(\mathcal{O}_{\Delta_{1}} / f \mathcal{O}_{\Delta_{1}}\right)^{*} / i\left((\mathbb{Z} / f \mathbb{Z})^{*}\right)$, where $i$ denotes the natural embedding of $\mathbb{Z} / f \mathbb{Z}$ into $\left(\mathcal{O}_{\Delta_{1}} / f \mathcal{O}_{\Delta_{1}}\right)^{*}$, as illustrated by the exact sequence (7.27) in [5, p.147].

Our reduction of the discrete logarithm problem in $C l\left(\Delta_{f}\right)$ to $C l\left(\Delta_{1}\right)$ and finite fields requires computing various preimages of elements in $\operatorname{Ker}\left(\phi_{C l}^{-1}\right)$ under the map $\psi$. Algorithm 1 (Std2Gen) accomplishes this task. The algorithm Reduce reduces an ideal $\mathfrak{A}$ given in standard representation and simultaneously computes a reducing number $\gamma \in \mathcal{O}_{\Delta_{1}}$ of the form $\left(\bar{x}+\bar{y} \sqrt{\Delta_{1}}\right) / 2$ such that $\mathfrak{A} / \gamma$ is reduced (see, for example, 14, Algorithm 2.6, p.16]).

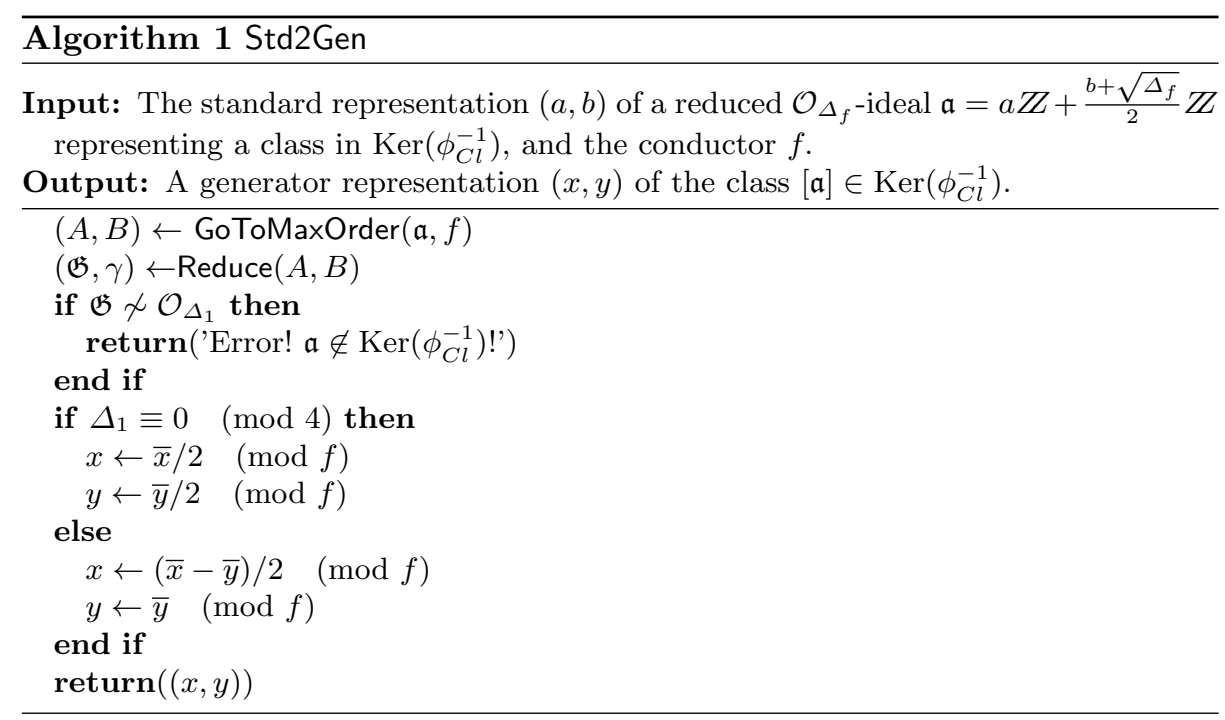

\section{The DLP for Arbitrary $C l\left(\Delta_{f}\right)$}

In this section we generalize the result from [12. We show that given the conductor $f$ and its prime factorization one can reduce the DLP in an arbitrary $C l\left(\Delta_{f}\right)$ to the DLP in various smaller groups. More precisely, we first show that the computation of discrete logarithms in $C l\left(\Delta_{f}\right)$ can be reduced to the computation of discrete logarithms in the class group $C l\left(\Delta_{1}\right)$ of the maximal order and the computation of discrete logarithms in $\operatorname{Ker}\left(\phi_{C l}^{-1}\right)$. Furthermore, we show that the latter problem boils down to the computation of discrete logarithms in a small number of finite fields. 
It should be noted that our method here is in essence a special case of the more general methods employed by Cohen et al. to compute discrete logarithms in ray class groups [3. The class group of a non-maximal order in any number field, not only degree 2, can be viewed as a ray class group of the maximal order, where the modulus is simply an integer, the conductor of the non-maximal order. Our exposition here is a reformulation of these results in terms of the simpler, special case of non-maximal orders using the language of 12. In addition, we prove that the reduction of the DLP in $C l\left(\Delta_{f}\right)$ to computing discrete logarithm computations in $C l\left(\Delta_{1}\right)$ and a small number of finite fields is of polynomial complexity.

We start with an algorithm which reduces the DLP in $C l\left(\Delta_{f}\right)$ to the DLP in $C l\left(\Delta_{1}\right)$ and $\operatorname{Ker}\left(\phi_{C l}^{-1}\right)$. Since the map $\psi:\left(\mathcal{O}_{\Delta_{1}} / f \mathcal{O}_{\Delta_{1}}\right)^{*} \rightarrow \operatorname{Ker}\left(\phi_{C l}^{-1}\right)$ given in Proposition 3 induces the isomorphism $\operatorname{Ker}\left(\phi_{C l}^{-1}\right) \cong\left(\mathcal{O}_{\Delta_{1}} / f \mathcal{O}_{\Delta_{1}}\right)^{*} / i\left((\mathbb{Z} / f \mathbb{Z})^{*}\right)$, we will reduce the latter DLP to computations in $\left(\mathcal{O}_{\Delta_{1}} / f \mathcal{O}_{\Delta_{1}}\right)^{*}$. Thus, our algorithm makes use of the following two methods:

- $\operatorname{DLPinCl}(\mathfrak{G}, \mathfrak{A})$

Accepts two reduced $\mathcal{O}_{\Delta_{1}}$-ideals $\mathfrak{G}, \mathfrak{A}$ as input and returns $x \in \mathbb{Z}$ with $0 \leq$ $x<h\left(\Delta_{1}\right)$ such that $\mathfrak{G}^{x} \sim \mathfrak{A}$, or $x=-1$ if no such $x$ exists.

- DLPinKerphi $\left(\gamma, \alpha,\left|\operatorname{Ker}\left(\phi_{C l}^{-1}\right)\right|\right)$

Accepts two generator representations $\gamma, \alpha$ of classes in $\operatorname{Ker}\left(\phi_{C l}^{-1}\right)$ such that $[\gamma],[\alpha] \in\left(\mathcal{O}_{\Delta_{1}} / f \mathcal{O}_{\Delta_{1}}\right)^{*}$ as input and returns $x \in \mathbb{Z}$ with $0 \leq x<\left|\operatorname{Ker}\left(\phi_{C l}^{-1}\right)\right|$ such that $\psi([\gamma])^{x}=\psi([\alpha])$ in $\operatorname{Ker}\left(\phi_{C l}^{-1}\right)$, or $x=-1$ if no such $x$ exists.

Furthermore, we assume that $h\left(\Delta_{1}\right)$ is known. This is no practical restriction, since the best currently known algorithm [1413] for computing discrete logarithms in $C l\left(\Delta_{1}\right)$ needs to compute $h\left(\Delta_{1}\right)$ and the group structure of $C l\left(\Delta_{1}\right)$ before the actual DL-computation starts. Secondly, if there were any other algorithm DLPinCl with the above properties, then one could use it to compute $h\left(\Delta_{1}\right)$, as shown in the full paper [10].

Algorithm 2 (ReduceDLP) reduces the DLP in $C l\left(\Delta_{f}\right)$ to the DLP in $C l\left(\Delta_{1}\right)$ and $\operatorname{Ker}\left(\phi_{C l}^{-1}\right) \stackrel{\sim}{=}\left(\mathcal{O}_{\Delta_{1}} / f \mathcal{O}_{\Delta_{1}}\right)^{*} / i\left((\mathbb{Z} / f \mathbb{Z})^{*}\right)$. The proof of correctness can be found in the full version of the paper [10].

Proposition 4. Given the conductor $f$, the class number $h\left(\Delta_{1}\right)$ and the order of the kernel $\left|\operatorname{Ker}\left(\phi_{C l}^{-1}\right)\right|$ one can reduce the DLP in $C l\left(\Delta_{f}\right)$ in $O\left(\log \left(\left|\Delta_{f}\right|\right)^{3}\right)$ bit-operations to the DLP in $C l\left(\Delta_{1}\right)$ and $\operatorname{Ker}\left(\phi_{C l}^{-1}\right)$.

Thus, in order to compute discrete logarithms in $C l\left(\Delta_{f}\right)$, we need efficient algorithms for computing discrete logarithms in $C l\left(\Delta_{1}\right)$ and $\operatorname{Ker}\left(\phi_{C l}^{-1}\right)$. The subexponential algorithm described in [13 Algorithm 3.3] is the most efficient algorithm known for computing discrete logarithms in $C l\left(\Delta_{1}\right)$. We now consider the $\mathrm{DLP}$ in $\operatorname{Ker}\left(\phi_{C l}^{-1}\right) \cong\left(\mathcal{O}_{\Delta_{1}} / f \mathcal{O}_{\Delta_{1}}\right)^{*} / i\left((\mathbb{Z} / f \mathbb{Z})^{*}\right)$ more closely.

By the Chinese Remainder Theorem (see, for example, [15, p.11]), the DLP in $\left(\mathcal{O}_{\Delta_{1}} / f \mathcal{O}_{\Delta_{1}}\right)^{*} / i\left((\mathbb{Z} / f \mathbb{Z})^{*}\right)$ boils down to DLPs in $\left(\mathcal{O}_{\Delta_{1}} / p_{i}^{e_{i}} \mathcal{O}_{\Delta_{1}}\right)^{*} / i\left(\left(\mathbb{Z} / p_{i}^{e_{i}} \mathbb{Z}\right)^{*}\right)$ for prime powers $p_{i}^{e_{i}}$, where $f=\prod p_{i}^{e_{i}}$. Furthermore, this problem can be efficiently reduced to the prime case $\left(\mathcal{O}_{\Delta_{1}} / p_{i} \mathcal{O}_{\Delta_{1}}\right)^{*} / i\left(\mathbb{F}_{p_{i}}^{*}\right)$. We give an algorithm (ReducePe2P) for this reduction in the full version of the paper [10]. 


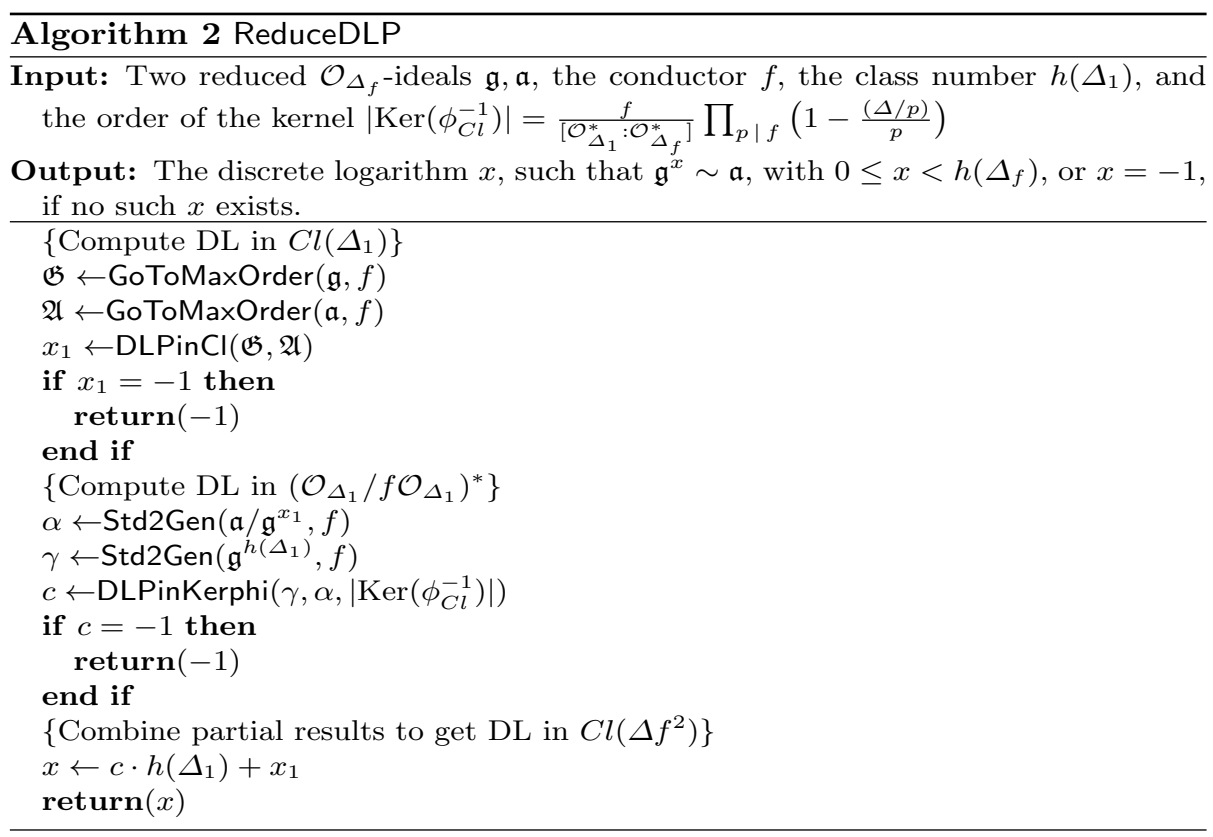

Proposition 5. The DLP in $\left(\mathcal{O}_{\Delta_{1}} / p^{e} \mathcal{O}_{\Delta_{1}}\right)^{*} / i\left(\left(\mathbb{Z} / p^{e} \mathbb{Z}\right)^{*}\right)$ can be reduced in $O(e$. $\left.\left(\log p^{e}\right)^{3}\right)$ bit-operations to $2 e$ DL-computations in $\left(\mathcal{O}_{\Delta_{1}} / p \mathcal{O}_{\Delta_{1}}\right)^{*} / i\left(\mathbb{F}_{p}^{*}\right)$.

Corollary 1. If $e=O\left((\log p)^{\alpha}\right)$ for some $\alpha=O(1)$, then the DLP in $\left(\mathcal{O}_{\Delta_{1}} / p^{e} \mathcal{O}_{\Delta_{1}}\right)^{*} / i\left(\left(\mathbb{Z} / p^{e} \mathbb{Z}\right)^{*}\right)$ can be reduced in polynomial time (in $\log p$ ) to the $\operatorname{DLP}$ in $\left(\mathcal{O}_{\Delta_{1}} / p \mathcal{O}_{\Delta_{1}}\right)^{*} / i\left(\mathbb{F}_{p}^{*}\right)$.

Using ReduceDLP and ReducePe2P allows us to reduce the DLP in $C l\left(\Delta_{f}\right)$ to DLPs in $C l\left(\Delta_{1}\right)$ and $\left(\mathcal{O}_{\Delta_{1}} / p \mathcal{O}_{\Delta_{1}}\right)^{*} / i\left(\mathbb{F}_{p}^{*}\right)$. As shown in 1211, $\left(\mathcal{O}_{\Delta_{1}} / p \mathcal{O}_{\Delta_{1}}\right)^{*}$ is isomorphic to either $\mathbb{F}_{p}^{*} \times \mathbb{F}_{p}^{*}$ or $\mathbb{F}_{p^{2}}^{*}$, depending how $p$ splits in $\mathcal{O}_{\Delta_{1}}$. This immediately leads to the central result of this section.

Theorem 1. If the prime factorization of the conductor $f=\prod_{i=1}^{k} p_{i}^{e_{i}}$ is known and $e_{i}=O\left(\left(\log p_{i}\right)^{\alpha}\right)$ for some $\alpha=O(1)$ then one can reduce the discrete logarithm problem in $C l\left(\Delta_{f}\right)$ in polynomial time (in $\log \Delta_{f}$ ) to the computation of logarithms in $C l\left(\Delta_{1}\right)$ and the following groups $(1 \leq i \leq k)$ :

$$
\begin{aligned}
& \mathbb{F}_{p_{i}}^{*}, \text { if }\left(\frac{\Delta_{1}}{p_{i}}\right) \in\{0,1\} \\
& \mathbb{F}_{p_{i}^{2}}^{*}, \text { if }\left(\frac{\Delta_{1}}{p_{i}}\right)=-1 .
\end{aligned}
$$

Proof. If the conductor $f$ and its prime factorization are known, then one can use ReduceDLP (Algorithm 2) to reduce the DLP in $C l\left(\Delta_{f}\right)$ to the DLP in $C l\left(\Delta_{1}\right)$ and $\operatorname{Ker}\left(\phi_{C l}^{-1}\right)$. By Proposition 4 this is possible in polynomial time in $\log \Delta_{f}$. By the Chinese Remainder Theorem (using the known factorization of $f$ ) the 
DLP in $\operatorname{Ker}\left(\phi_{C l}^{-1}\right) \stackrel{\simeq}{=}\left(\mathcal{O}_{\Delta_{1}} / f \mathcal{O}_{\Delta_{1}}\right)^{*} / i\left((\mathbb{Z} / f \mathbb{Z})^{*}\right)$ is nothing more than the DLP in groups of the form $\left(\mathcal{O}_{\Delta_{1}} / p_{i}^{e_{i}} \mathcal{O}_{\Delta_{1}}\right)^{*} / i\left(\left(\mathbb{Z} / p_{i}^{e_{i}} \mathbb{Z}\right)^{*}\right)$, which can, using ReducePe2P (from [10]) and Corollary [1, be reduced in polynomial time (in $\log p_{i}$ ) to the DLP in $\left(\mathcal{O}_{\Delta_{1}} / p_{i} \mathcal{O}_{\Delta_{1}}\right)^{*} / i\left(\mathbb{F}_{p_{i}}^{*}\right)$, because $e_{i}$ is assumed to be polynomial in $\log p_{i}$.

It remains to show how one reduces the discrete logarithm problem in $\left(\mathcal{O}_{\Delta_{1}} / p \mathcal{O}_{\Delta_{1}}\right)^{*} / i\left(\mathbb{F}_{p}^{*}\right)$ to discrete logarithm problems in $\mathbb{F}_{p}^{*}$ or $\mathbb{F}_{p^{2}}^{*}$. Suppose we have two representatives $\gamma, \alpha$ of classes in $\left(\mathcal{O}_{\Delta_{1}} / p \mathcal{O}_{\Delta_{1}}\right)^{*}$ for which we want to compute the discrete logarithm $c$ such that $[\gamma]^{c} \equiv[\alpha]$ in $\left(\mathcal{O}_{\Delta_{1}} / p \mathcal{O}_{\Delta_{1}}\right)^{*} / i\left(\mathbb{F}_{p}^{*}\right)$. In the inert case $\left(\Delta_{1} / p\right)=-1$, where $\left(\mathcal{O}_{\Delta_{1}} / p \mathcal{O}_{\Delta_{1}}\right)^{*} \cong \mathbb{F}_{p^{2}}^{*}$, we have $\left(\mathcal{O}_{\Delta_{1}} / p \mathcal{O}_{\Delta_{1}}\right)^{*} / i\left(\mathbb{F}_{p}^{*}\right) \cong \mathbb{F}_{p^{2}}^{*} / i\left(\mathbb{F}_{p}^{*}\right)$. It is well-known that there always exists a surjective homomorphism from $\mathbb{F}_{p^{2}}^{*}$ to $\mathbb{F}_{p^{2}}^{*} / i\left(\mathbb{F}_{p}^{*}\right)$. Thus, we first solve the DLP $\gamma^{c^{\prime}} \equiv \alpha \quad\left(\bmod p \mathcal{O}_{\Delta_{1}}\right)$ by simply solving the corresponding DLP in $\mathbb{F}_{p^{2}}^{*}$. Taking $c \equiv c^{\prime} \bmod (p+1)$ yields the required solution to the DLP $[\gamma]^{c} \equiv[\alpha]$ in $\left(\mathcal{O}_{\Delta_{1}} / p \mathcal{O}_{\Delta_{1}}\right)^{*} / i\left(\mathbb{F}_{p}^{*}\right)$.

We now restrict our attention to the split case $\left(\Delta_{1} / p\right)=1$, where we have $\left(\mathcal{O}_{\Delta_{1}} / p \mathcal{O}_{\Delta_{1}}\right)^{*} \cong \mathbb{F}_{p}^{*} \times \mathbb{F}_{p}^{*}$. The element $\gamma=\left(x_{1}, y_{1}\right)$ maps to $\left(x_{1} \bmod p, y_{1} \bmod \right.$ $p) \in \mathbb{F}_{p}^{*} \times \mathbb{F}_{p}^{*}$ and similarly $\alpha=\left(x_{2}, y_{2}\right) \operatorname{maps}$ to $\left(x_{2} \bmod p, y_{2} \bmod p\right)$. The DLP in $\left(\mathcal{O}_{\Delta_{1}} / p \mathcal{O}_{\Delta_{1}}\right)^{*} / i\left(\mathbb{F}_{p}^{*}\right)$ becomes

$$
\left(x_{1}, y_{1}\right)^{c} \equiv l\left(x_{2}, y_{2}\right) \quad\left(\text { in } \mathbb{F}_{p}^{*} \times \mathbb{F}_{p}^{*}\right)
$$

which in turn yields the simultaneous DLP's

$$
x_{1}^{c} \equiv l x_{2} \quad(\bmod p), \quad y_{1}^{c} \equiv l y_{2} \quad(\bmod p) .
$$

Since these two DLP's must be solved for the same $c$ and $l$, we can combine them and obtain the single DLP in $\mathbb{F}_{p}^{*}$

$$
\left(\frac{x_{1}}{y_{1}}\right)^{c} \equiv\left(\frac{x_{2}}{y_{2}}\right) \quad(\bmod p)
$$

from which we can find the desired value of $c$.

As noted in [8], this simple strategy can be used to improve the general maps from [12 11]; it is shown that in this case there not only exists a surjective homomorphism $\mathbb{F}_{p}^{*} \times \mathbb{F}_{p}^{*} \rightarrow \operatorname{Ker}\left(\phi_{C l}^{-1}\right)$, but even an efficiently computable isomorphism $\mathbb{F}_{p}^{*} \cong \operatorname{Ker}\left(\phi_{C l}^{-1}\right)$.

Note that the central result of [12] now is nothing more than an immediate corollary.

\subsection{Example}

We illustrate the reduction of discrete logarithm computations in $C l\left(\Delta_{f}\right)$ via a small example. Suppose $\Delta_{1}=-1019, f=23$, and $\Delta_{f}=\Delta_{1} f^{2}=-539051$. In this case, both $C l\left(\Delta_{f}\right)$ and $C l\left(\Delta_{1}\right)$ are cyclic with $h\left(\Delta_{1}\right)=13$ and $h\left(\Delta_{f}\right)=$ $h\left(\Delta_{1}\right)(23-1)=286$. The equivalence class represented by the reduced ideal

$$
\mathfrak{g}=15 \mathbb{Z}+\frac{-7+\sqrt{-539051}}{2} \mathbb{Z}=(15,-7)
$$


generates $C l\left(\Delta_{f}\right)$.

Suppose we wish to compute the discrete logarithm of $[\mathfrak{a}]$ with respect to the base $[\mathfrak{g}]$ in $C l\left(\Delta_{f}\right)$, where

$$
\mathfrak{a}=11 \mathbb{Z}+\frac{9+\sqrt{-539051}}{2} \mathbb{Z}=(11,9) .
$$

That is, we want to find $x$ such that $\mathfrak{g}^{x} \sim \mathfrak{a}$. Since $\mathfrak{g}$ generates $C l\left(\Delta_{f}\right)$, we know that such an $x$ exists. Following ReduceDLP (Algorithm 2), we first compute $[\mathfrak{G}]=\left[\phi_{C l}^{-1}(\mathfrak{g})\right]$ and $[\mathfrak{A}]=\left[\phi_{C l}^{-1}(\mathfrak{a})\right]$, and solve the discrete logarithm problem

$$
\mathfrak{G}^{x_{1}} \sim \mathfrak{A}
$$

in $C l\left(\Delta_{1}\right)$. We have $\mathfrak{G}=15 \mathbb{Z}+\frac{1+\sqrt{-1019}}{2} \mathbb{Z}=(15,1), \mathfrak{A}=(11,9)$, and we easily compute $x_{1}=9$.

At this point we know that $x$ has the form $x=c \cdot h\left(\Delta_{1}\right)+x_{1}=13 c+9$, and it remains to compute $c$. Again following ReduceDLP (Algorithm 2), we compute generator representations $\alpha, \gamma$ of $[\alpha],[\gamma] \in\left(\mathcal{O}_{\Delta_{1}} / f \mathcal{O}_{\Delta_{1}}\right)^{*}$ such that $\psi([\alpha])=\left[\mathfrak{a} / \mathfrak{g}^{x_{1}}\right]$ and $\psi([\gamma])=\left[\mathfrak{g}^{h\left(\Delta_{1}\right)}\right]$. Following Std2Gen (Algorithm 1), we first compute

$$
\mathfrak{b} \sim \mathfrak{a} / \mathfrak{g}^{x_{1}} \sim \mathfrak{a} / \mathfrak{g}^{9}=(311,277)
$$

and

$$
\mathfrak{c} \sim \mathfrak{g}^{h\left(\Delta_{1}\right)} \sim \mathfrak{g}^{13}=(297,295) .
$$

To find $\alpha$ and $\gamma$ we compute the principal ideals $\mathfrak{B}=\varphi^{-1}(\mathfrak{b})$ and $\mathfrak{C}=\varphi^{-1}(\mathfrak{c})$, and reduce them while simultaneously computing their modulo $f \mathcal{O}_{\Delta_{1}}$ reduced generators, which we take as $\alpha$ and $\gamma$. We obtain $\mathfrak{B}=(311,-15)=(\alpha)$ and $\mathfrak{C}=(297,-13)=(\gamma)$ where

$$
\alpha=-8+1 \omega, \quad \gamma=-7+1 \omega
$$

and $\omega=\frac{1+\sqrt{-1019}}{2}$.

To compute $c$, we need to solve the discrete logarithm problem

$$
[\gamma]^{c} \equiv[\alpha] \quad\left(\text { in } \operatorname{Ker}\left(\phi_{C l}^{-1}\right) \cong\left(\mathcal{O}_{\Delta_{1}} / f \mathcal{O}_{\Delta_{1}}\right)^{*} / i\left((\mathbb{Z} / f \mathbb{Z})^{*}\right)\right) .
$$

For this example, we have $\left(\Delta_{1} / f\right)=(-1019 / 23)=1$, and thus $\left(\mathcal{O}_{\Delta_{1}} / f \mathcal{O}_{\Delta_{1}}\right)^{*} \simeq$ $\mathbb{F}_{23}^{*} \times \mathbb{F}_{23}^{*}$ by [12 Lemma 8]. Since $\omega \equiv 14 \quad(\bmod 23)$ and $\bar{\omega} \equiv 10 \quad(\bmod 23)$, we obtain

$$
\gamma \mapsto(-7+1 \omega \bmod 23,-7+1 \bar{\omega} \bmod 23)=(7,3) \in \mathbb{F}_{23}^{*} \times \mathbb{F}_{23}^{*}
$$

and

$$
\alpha \mapsto(-8+1 \omega \bmod 23,-8+1 \bar{\omega} \bmod 23)=(6,2) \in \mathbb{F}_{23}^{*} \times \mathbb{F}_{23}^{*} .
$$

Since $\operatorname{Ker}\left(\phi_{C l}^{-1}\right) \cong\left(\mathbb{F}_{p}^{*} \times \mathbb{F}_{p}^{*}\right) / i\left(\mathbb{F}_{p}^{*}\right)$, we need to find $c$ by solving the discrete logarithm problem $(7,3)^{c}=l(6,2)$ in $\mathbb{F}_{23}^{*} \times \mathbb{F}_{23}^{*}$ for every $l \in \mathbb{F}_{23}^{*}$. This yields

$$
7^{c} \equiv 6 l \quad(\bmod 23), \quad 3^{c} \equiv 2 l \quad(\bmod 23),
$$


and we combine these two discrete logarithm problems to obtain one discrete logarithm problem in $\mathbb{F}_{23}^{*}$ :

$$
(7 / 3)^{c} \equiv(6 / 2) \quad(\bmod 23) \rightarrow 10^{c} \equiv 3 \quad(\bmod 23) .
$$

Solving yields $c=20$, and finally $x=13 \cdot 20+9=269$. It is easy to verify that $x$ is indeed the desired discrete logarithm: simply compute the reduced ideal $\mathfrak{g}^{269}$ and verify that it is equal to the reduced ideal $\mathfrak{a}$.

\section{Towards Practical Non-interactive Cryptosystems}

Before we explain our system setup we list the crucial properties:

\section{Required Properties}

1. The discrete logarithm problem (DLP) in $C l\left(\Delta_{p}\right)$ without knowing the factorization of $\Delta_{p}=\Delta_{1} p^{2}$ is infeasible. To determine bounds for $\Delta_{1}$ and $p$, we make use of the heuristic model from [7], which is a refinement of Lenstra and Verheul's approach [16, since it also takes into account the asymptotically vanishing $o(1)$-part in subexponential algorithms. We will now derive bounds for the parameters such that an attacker would need to spend about 90,000 MIPS years to break the system. This approximately amounts to a ten-fold higher workload than the recent factorization of RSA155 and hence corresponds to the very minimum requirements. The estimates in [7, Table 3] state that $\Delta_{p}$ should have at least $576,667,423$ bits to prevent factoring $\Delta_{p}$ with the GNFS, factoring $\Delta_{p}$ with ECM and computing discrete logarithms in $C l\left(\Delta_{p}\right)$ with the SIQS-analogue [14, respectively.

$1.1 \Delta_{p}$ is large enough that using the subexponential algorithm from 13 to directly compute discrete logarithms in $C l\left(\Delta_{p}\right)$ is infeasible. $\Delta_{p}>2^{423}$ implies an expected workload of more than 90,000 MIPS years.

$1.2 \Delta_{p}$ cannot be factored to reduce the DLP to DLPs in $C l\left(\Delta_{1}\right)$ and $\mathbb{F}_{p}^{*}$ (or $\left.\mathbb{F}_{p^{2}}^{*}\right)$.

1.2.1 $\Delta_{p}$ is large enough so that the Number Field Sieve would need more than 90,000 MIPS years. This yields $\Delta_{p}>2^{576}$.

1.2.2 $\Delta_{1}$ and $p$ are large enough that it would take more than 90,000 MIPS years to find them with the Elliptic Curve Method. This implies $\Delta_{1}, p>2^{222}$.

2. $\Delta_{1}, p$ must be small enough to enable the KGC to compute discrete logarithms in $C l\left(\Delta_{1}\right)$ and $\mathbb{F}_{p}^{*}$ using subexponential algorithms. $\Delta_{1}, p<2^{300}$ seems to be feasible.

3. $\mathrm{Cl}\left(\Delta_{p}\right)$ must be cyclic.

It is easy to see that the following setup satisfies all above requirements. 


\section{System Setup}

1. The KGC randomly chooses a prime $q \equiv 3 \quad(\bmod 4), q>2^{260}$, sets $\Delta_{1}=-q$ and computes $h\left(\Delta_{1}\right)$ and the group structure of $C l\left(\Delta_{1}\right)$ with the algorithm from 14. The Cohen-Lenstra heuristics 4 suggest that $C l\left(\Delta_{1}\right)$ is cyclic with probability $>0.97$. If $C l\left(\Delta_{1}\right)$ is not cyclic, the KGC selects another prime $q$ until it is cyclic.

2. The KGC chooses a prime $p>2^{260}$ with $\left(\Delta_{1} / p\right)=1$ and $\operatorname{gcd}\left(p-1, h\left(\Delta_{1}\right)=1\right.$ such that the SNFS can be applied as in [24, and computes $\Delta_{p}=\Delta_{1} p^{2}$. The gcd condition ensures that $C l\left(\Delta_{p}\right)$ is cyclic.

3. The KGC computes a generator $\mathfrak{g}$ of $C l\left(\Delta_{p}\right)$ and publishes it together with $\Delta_{p}$

Given a generator $\mathfrak{G}$ of $C l\left(\Delta_{1}\right)$, which the KGC can easily obtain during the computation of $C l\left(\Delta_{1}\right)$ [14, Algorithm 6.1], it is also easy in practice to find a generator $\mathfrak{g}$ of $C l\left(\Delta_{p}\right)$ with the additional property that $\phi_{C l}^{-1}(\mathfrak{g})=\mathfrak{G}$. The KGC repeatedly selects random values of $\alpha \in \mathcal{O}_{\Delta_{1}}$ and takes the first $\mathfrak{g}=\phi(\alpha \mathfrak{G})$ such that $\mathfrak{g}^{h\left(\Delta_{p}\right) / d_{i}} \not \mathcal{O}_{\Delta_{p}}$ for any positive divisor $d_{i}$ of $h\left(\Delta_{p}\right)$. Although $h\left(\Delta_{p}\right)$ is approximately as large as $\sqrt{\left|\Delta_{p}\right|}$, in practice it has sufficiently many small factors that this condition can be verified with high probability.

\section{User Registration}

1. Bob requests the public key $\mathfrak{b}$ corresponding to his identity $I D_{B}$ at the KGC.

2. The KGC verifies Bob's identity, for example, using a passport, and starts with the key generation.

3. The KGC computes the 128-bit hash $i d=h\left(I D_{B}\right)$ using, for example, MD5 [22], of Bob's identity and embeds $i d$ into a group element of $C l\left(\Delta_{p}\right)$ by taking the largest prime $p_{B} \leq i d$, for which $\left(\Delta_{p} / p_{B}\right)=1$ and computing the prime ideal $\mathfrak{b}=p_{B} \mathbb{Z}+\frac{b_{B}+\sqrt{\Delta_{p}}}{2}$, where $b_{B}$ is the uniquely determined square root of $\Delta_{p} \bmod 4 p_{B}$ with $0 \leq b_{B} \leq p_{B}$. Note that $\mathfrak{b}$ is already reduced, since $\sqrt{\left|\Delta_{p}\right|}>2^{128}>p_{B}$. If the KGC recognizes that $\mathfrak{b}$ is already assigned to another user it will ask Bob to choose another identity, for example, his postal address.

4. Finally, the KGC computes the discrete logarithm $b$ such that $\mathfrak{g}^{b} \sim \mathfrak{b}$ using the secret knowledge of the conductor $p$ and the reduction procedure described in the Section 3, and returns $b$ to Bob.

As soon as all users are registered this way the KGC can destroy the factorization of $\Delta_{p}$ and cease to exist. The users can obtain any other user's authentic public key simply by hashing that user's identity and computing the largest prime ideal whose norm is less than the hash value. Each user has a public/private key-pair (a, $a$ ) with $\mathfrak{a} \sim \mathfrak{g}^{a}$, so discrete logarithm-based protocols such as Diffie-Hellman or ElGamal can be directly applied in the class group $C l\left(\Delta_{p}\right)$. 
Preliminary experiments, together with computational experience using the subexponential algorithms from [13] and 24], indicate that a KGC with modest computational resources, for example, a small network of Pentium processors, should be able to set up a key distribution system using $p, q \approx 2^{300}$ at least. For such an example, we estimate that after a precomputation of about 3 days on a cluster of $16550 \mathrm{Mhz}$ Pentiums III's for computing the class group $C l\left(\Delta_{1}\right)$, each user registration would take about 1 day on a single $550 \mathrm{Mhz}$ Pentiums III, the vast majority of this time being spent on the computation of discrete logarithms in $\mathbb{F}_{p}^{*}$. However, adding more machines to the cluster yields a linear speedup in both the precomputation stage and part of the user registration stage. Thus, although this level of complexity is far from ideal, unlike the case of $(\mathbb{Z} / n \mathbb{Z})^{*}$ it is at least possible to set up noninteractive systems with secure parameters in $C l\left(\Delta_{p}\right)$. More detailed computational results will appear in the full version of the paper [10].

\section{References}

1. Z.I. Borevich and I.R. Shafarevich. Number Theory. Academic Press, New York, 1966 .

2. H. Cohen. A Course in Computational Algebraic Number Theory. Springer-Verlag, Berlin, 1993.

3. H. Cohen, F. Diaz y Diaz, and M. Olivier. Computing ray class groups, conductors, and discriminants. Math. Comp., 67(222):773-795, 1998.

4. H. Cohen and H.W. Lenstra, Jr. Heuristics on class groups of number fields. In Number Theory, Lecture notes in Math., volume 1068, pages 33-62. SpringerVerlag, New York, 1983.

5. D.A. Cox. Primes of the form $x^{2}+n y^{2}$. John Wiley \& Sons, New York, 1989.

6. D. Hühnlein. Efficient implementation of cryptosystems based on non-maximal imaginary quadratic orders. In Selected Areas in Cryptography - SAC'99, volume 1758 of $L N C S$, pages 150-167, 1999.

7. D. Hühnlein. Quadratic orders for NESSIE - Overview and parameter sizes of three public key families. Technical report TI 03/00, TU-Darmstadt, 2000

8. D. Hühnlein. Faster generation of NICE-Schnorr signatures. in preparation, 2000

9. D. Hühnlein, M.J. Jacobson, Jr., S. Paulus, and T. Takagi. A cryptosystem based on non-maximal imaginary quadratic orders with fast decryption. In Advances in Cryptology - EUROCRYPT '98, volume 1403 of LNCS, pages 294-307, 1998.

10. D. Hühnlein, M.J. Jacobson, and D. Weber. Towards practical non-interactive public key cryptosystems using non-maximal imaginary quadratic orders. CORR technical report, www.cacr.math.uwaterloo.ca, to appear.

11. D. Hühnlein and J. Merkle. An efficient NICE-Schnorr-type signature scheme. In Proceedings of Public Key Cryptography 2000, Springer, volume 1751 of LNCS, pages $14-27,2000$.

12. D. Hühnlein and T. Takagi. Reducing logarithms in totally non-maximal imaginary quadratic orders to logarithms in finite fields. In Advances in Cryptology ASIACRYPT '99, volume 1716 of LNCS, pages 219-231, 1999.

13. M.J. Jacobson, Jr. Computing discrete logarithms in quadratic orders. To appear Journal of Cryptology, 1999. 
14. M.J. Jacobson, Jr. Subexponential Class Group Computation in Quadratic Orders. PhD thesis, Technische Universität Darmstadt, Darmstadt, Germany, 1999.

15. S. Lang. Algebraic number theory. Springer, Berlin, 2nd edition, 1991. ISBN 3-540-94225-4.

16. A.K. Lenstra, E. Verheul. Selecting Cryptographic Key Sizes. In Proceedings of Public Key Cryptography 2000, Springer, volume 1751 of LNCS, pages 446-465, 2000.

17. C.H. Lim and P.J. Lee. Modified Maurer-Yacobi's scheme and its applications. In Proceedings of Auscrypt'92, LNCS, pages 308-323, 1992.

18. M. Maurer and D. Kügler. A note on the weakness of the Maurer-Yacobi squaring method. Technical report, TI 15/99, TU Darmstadt, 1999.

19. U. Maurer and Y. Yacobi. Non-interactive public-key cryptography. In Advances in Cryptology - EUROCRYPT'91, volume 547 of LNCS, pages 498-507, 1991.

20. U. Maurer and Y. Yacobi. A remark on a non-interactive public-key distribution system. In Advances in Cryptology - EUROCRYPT'92, volume 658 of LNCS, pages 458-460, 1993.

21. U. Maurer and Y. Yacobi. A non-interactive public-key distribution system. Design Codes and Cryptography, 9:305-316, 1996.

22. R. Rivest. The MD5 message-digest algorithm, 1992. RFC1321, Internet Activities Board, Internet Engineering Task Force.

23. A. Shamir. Identity based cryptosystems and signature schemes. In Advances in Cryptology - CRYPTO '84, volume 196 of LNCS, pages 47-53, 1985.

24. D. Weber and T. Denny. The solution of McCurley's discrete log challenge. In Advances in Cryptology - CRYPTO '98, volume 1462 of LNCS, pages 56-60, 1998. 